\title{
Variation in the Thickness of a Fluid Interface Due to Internal Wave Propagation: A Lattice Boltzmann Simulation
}

\author{
${ }^{1}$ J.M. Buick, ${ }^{2}$ D.B. Hann and ${ }^{2}$ J.A. Cosgrove \\ ${ }^{1}$ Department of Physics and Electronics, \\ School of Biological, Biomedical and Molecular Sciences \\ University of New England, Armidale, NSW 2351, Australia \\ ${ }^{2}$ Department of Physics and Astronomy, the University of Edinburg, Mayfield Road \\ Edinburgh EH9 3JZ, UK
}

\begin{abstract}
The change in the thickness of an interface between two immiscible fluids due to the propagation of an internal capillary-gravity wave along the interface is considered using a Bhatnagar, Gross and Krook (BGK) lattice Boltzmann model of a binary of fluid. The vertical thickness of the interface is recorded from the simulations since this is the most easily measured quantities in any simulation or experiment. The vertical thickness is then related to the actual thickness (perpendicular to the interface) which is seen to vary with the phase of the wave. The positions of the maxima and minimum thicknesses are seen to be approximately constant relative to the phase of the propagating wave and the range of variation of the thickness decreases at approximately the same rate as the wave amplitude is damped. A simplified model for the interface is considered which predicts a similar variation due to the interface being stretched as the internal wave propagates.
\end{abstract}

Key words: Lattice Boltzmann Model, Thickness, Fluid Interface

\section{INTRODUCTION}

Internal waves can propagate along an interface between fluids of similar densities in the same manner as surface waves propagate along the interface between a liquid and a gas, as is most commonly observed between water and air. Internal waves vary in scale from capillary waves with wavelengths of the order of $10^{-7} \mathrm{~m}^{[1]}$ to gravity waves with wavelengths of the order of $10^{2} \mathrm{~m}^{[2,3]}$. Capillary waves have wavelengths which are short enough that gravity forces are negligible compared to the surface tension forces acting at the interface, and can occur between any two immiscible fluids. Internal gravity is dominant over surface tension for the waves have a wavelength large enough that gravity is dominant over surface tension for the wave motion. Internal gravity and not surface tension is responsible for driving the motion, they occur whenever there is a change in the specific density of the fluid, either at the interface between two fluids of different densities such as fresh and salt water, or where the density of a fluid change rapidly such as at a thermo Cline. At intermediate wavelengths both gravity and surface tension have a significant effect and capillarygravity waves are observed. The wavelengths at which the different regime of pure gravity, capillary-gravity and strength of the surface tension at the interface and the densities of the two fluids.

The shape of internal waves has been studied by many authors, ${ }^{[4,6]}$. Here we consider the manner in which an interface between two fluids is altered due to wave propagation at the interface, and in particular we examine this by studying the change which occurs in the thickness of the interface. A numerical study is performed using an immiscible binary fluid consisting of two fluids of similar but distinct specific densities. The parameters used in this study have been selected so that both gravitational and surface tension forces are responsible for the wave motion.

\section{MATERIALS AND METHODS}

Numerical Model: The internal waves are simulated using a lattice Boltzmann model which has been used previously to model internal standing gravity and capillary-gravity wave ${ }^{[7,8]}$ and progressive waves ${ }^{[8]}$. This utilizes the immiscible binary fluid BGK model proposed by ${ }^{[9,10]}$ with the inclusion of a body force $e^{[7]}$. Rather than considering separately the two density components of the binary fluid, $\rho 1$ and $\rho_{2}$, we work with the total fluid density, $\rho=\rho_{1}+\rho_{2}$, and the concentration difference or order parameter, $d=\rho_{1}-\rho_{2}$. To this end we need to consider two BGK Boltzmann equations ${ }^{[11]}$ which describe the evolution of two distribution function $\mathrm{f}_{\mathrm{i}}$ and $\mathrm{g}_{\mathrm{i}}$ :

$$
f_{i}\left(\mathbf{r}+\mathbf{e}_{i}, t+1\right)-f_{i}(\mathbf{r}, t)=-\frac{1}{\tau_{f}}\left(f_{i}-\bar{f} i\right)+\frac{1}{3} \mathrm{~F}_{a} e_{i a}
$$

And:

$g_{i}\left(\mathbf{r}+\mathbf{e}_{i}, t+1\right)-g_{i}(\mathbf{r}, t)=-\frac{1}{\tau_{g}}\left(g_{i}-\overline{g_{i}}\right)$ 
where, $e_{i}$ is a unit vector along direction $i$ of the underlying lattice and $\tau \mathrm{f}$ and $\tau \mathrm{g}$ are three parameters which are considered later. These equations are the standard form of the lattice Boltzmann equation, with the addition of the term $\mathrm{F}_{\mathrm{a}} \mathrm{e}_{\mathrm{ia}} / 3$ which are associated with gravity force. Each equation describes the evolution of seven. Distribution functions, fi or gi, where $i=0$ represents the rest distribution function which remains stationary at a grid site and $i=1,2, \ldots$, 6 represent the distribution functions hexagonal grid on which the simulations are performed. The left hand sides of the Boltzmann equations correspond to the streaming of the distribution functions along the grid while the right hand side is a BGK collision operator ${ }^{[11]}$ which accounts for, in a simplified manner, the redistribution of the distribution functions due to particle collisions. The BGK collision operator of the

fluid to its equilibrium state $\left(\bar{f}_{i}\right.$ or $\left.\bar{g}_{i}\right)$ at a rate determined by the relaxation parameters $\tau_{\mathrm{f}}$ and $\tau_{\mathrm{g}}$. From the evolution of the distribution functions the macroscopic quantities can be obtained. The total fluid density, $\rho=\rho_{1} \rho_{2}$, the total fluid velocity, $u$, and the density difference, $d=\rho_{1} \rho 2$, can be found from the distribution functions as:

$$
\rho=\sum_{i} f_{i}, \quad \rho v_{\alpha}=\sum_{i} f_{i} e_{i a} \text { and } d=\sum_{i} g_{i}
$$

where, the summation is over all the lattice directions, from $\mathrm{i}=0$ to $\mathrm{i}=6$, and we use Greek subscripts to represent functions, $\bar{f}_{i}$ and $\bar{g}_{i}$ are then selinteractionsimulate two ideal gases with repulsive interactin energy ${ }^{[9,10]}$ and are given by:

$$
\begin{aligned}
& \bar{f}_{i}=A=B u_{\partial} e_{i a}=C u^{2}=D u_{a} u_{\beta} e_{i a} e_{i \beta}=G_{a \beta} e_{i a} e_{i \beta}, \text { for } i= \\
& 1,2, \ldots, 6 ; \text { and } \bar{f}_{0}=\mathrm{A}_{0}+\mathrm{C}_{0} u^{2}
\end{aligned}
$$

And:

$$
\begin{aligned}
& \bar{g}_{i}=H+K u_{a} e_{i a}+J u^{2}+Q u_{a} u_{\beta} e_{i a} e_{i \beta}, \text { for } i=1,2, \ldots, 6 \\
& \text { and } \bar{g}_{0}=H_{0}+J_{0} u^{2}
\end{aligned}
$$

Where:

$$
\begin{aligned}
& A=\left(\rho T-\kappa \nabla^{2} \frac{\rho}{2}\right) / 3, A_{0}=\rho-6 A \\
& B=\frac{\rho}{3}, C=-\frac{\rho}{6}, C_{0}=-\rho, D=\frac{2 \rho}{3} \\
& G_{x y}=G_{y x}=\frac{2 \kappa}{3}\left[\frac{\partial \rho}{\partial x} \frac{\partial \rho}{\partial y}+\frac{\partial d}{\partial x} \frac{\partial d}{\partial y}\right], G_{x x}=-G_{y y}=\frac{2 \kappa}{3} \\
& {\left[\left(\frac{\partial \rho}{\partial x}\right)^{2}-\left(\frac{\partial \rho}{\partial y}\right)^{2}+\left(\frac{\partial d}{\partial x}\right)^{2}-\left(\frac{\partial d}{\partial y}\right)^{2}\right]}
\end{aligned}
$$

$$
H=\frac{\Gamma \mu}{3}, H_{0}=-d-6 H, K \frac{d}{3}, J=\frac{d}{6}, J_{0}=-d, Q=\frac{2 d}{3} .
$$

The body force term and the chemical potential differences are given by:

$F=\left[\gamma_{a}(\rho-d)+\gamma b(\rho+d)\right] \hat{\mathrm{e}}_{z}$

And $\mu=-\frac{\Lambda d}{2 \rho}+\frac{T}{2} \log \left(\frac{1+d / \rho}{1-d / \rho}\right)-\kappa \nabla^{2} d$ respectively where $\hat{\mathrm{e}}_{z}$ is a unit vector in the vertical direction. The parameters which have not yet been defined can in general be selected to determine the properties of the simulation, ${ }^{[7,9,10]}$. Here we define them and give the value used here and, for the parameters of particular importance to our simulation, we briefly discuss their significance. The temperature $\mathrm{T}$ was set to 0.5 , the interaction strength parameter $\Lambda$ was set to 1.1 and the mobility $\Gamma$ was set to 0.1 ; for these parameters an immiscible binary fluid is simulated. The interfacial thickness of about ten lattice units. The gravitational coefficients $\gamma_{\mathrm{a}}$ and $\gamma_{\mathrm{b}}$ were set to $5.0 \times 10^{-5}$ and $5.5 \times 10^{-}$ 5 respectively; this gives a gravitational strength of $1.075 \times 10^{-4}$ and a relative density difference $=1.05$. The relaxation parameter $\tau_{f}$ is set to 0.7 ; this gives a fluid viscosity of 0.05 . The other relaxation parameter $\tau_{g}$ is set to 0.789 to eliminate third order corrections in the equation of motion for the order parameter. All these parameters are measured in the units of the lattice, that is the lattice spacing and the time-step. Comparison with a physical situation can be made by considering the dimension parameters describing the system such as the Froude number, the Reynolds number and the relative density difference. This binary fluid system can be shown ${ }^{[10]}$ to satisfy the continuity equation, $\partial_{t} \rho+\partial_{a} \rho u_{a}=0, \quad$ and the Navier-Stokes equation, $\partial_{t} \rho u_{a}=\partial_{\beta} \rho u_{a} u_{\beta}=-\partial_{a} p+v \partial_{\beta} \partial_{\beta} \rho u_{a}+\partial_{a} \varsigma \partial_{\beta} \rho u_{\beta}$, where $v=\frac{2 \tau_{f}-1}{8}$ and $\varsigma=\left(\tau_{f}-\frac{1}{2}\right)\left(\frac{1}{2}-\frac{\partial \rho}{\partial \rho}\right)$. This ensures that the lattice Boltzmann model used here is mimicking a real fluid. While the form of $\bar{f}_{i}$ and $\bar{g}_{i}$ ensure that the equilibrium state is thermodynamically consistent and a true binary fluid is being simulated.

Internal Wave Simulations: This lattice Boltzmann model was used to simulated progressive internal waves on an interface at the center of a 256 by 256 site grid. A solid no-slip boundary ${ }^{[12]}$ was applied at the bottom and top of the grid and periodic boundary conditions at the outer edges. The wave was initialized in four steps: (1) the grid is set-up with a horizontal interface and constant total density everywhere. Gravity is then applied to a steady-state is reached-this initializes the density is each fluid. (2) The interface is perturbed to a 
sinusoidal shape with wavelength $\lambda=256-$ this represents a standing wave at the extreme of its oscillation $^{[7]}$. (3) The standing wave is allowed to oscillate for a quarterly period until the interface is flat ${ }^{[7,13]}$ this allows the shape and width of the interface to form. (4) The velocities under the standing wave are measured and used to initialize the progressive wave, the shape of the interface to form. (4) The velocities under the standing wave are measured and used to initialize the progressive wave, the shape of the interface is formed by shifting the grid by an amount $\eta$, the shape of the interface ${ }^{[13]}$ this initializes the progressive wave with the correct velocities and interface shape (measured from the standing wave simulation) rather than imposing them on the wave. Using this initialization technique the vertical thickness of the interface is approximately constant. This technique is used so that no velocities or densities are imposed on the wave; alternatively the wave could be initialized from linear or higher-order wave theory.

Approximating the fluid by two immiscible, in viscid fluids at a sharp interface the frequency of the wave motion is given by ${ }^{[14,15]}$ :

$$
\omega^{2}=g^{\prime} k+\frac{k^{3} \sigma}{\rho(f+1)}
$$

where, $\sigma$ is the surface tension, $\mathrm{k}$ is the wave number, $\mathrm{f}$ is the relative density differences $\rho_{1} / \rho_{2}$ and g' is the relative gravity:

$g^{\prime}=\frac{f-1}{f+1} g$

Now, the strength of the surface tension can be determined by considering, in the absence of gravity, a 'bubble' of one fluid inside the second. The density difference, $\Delta \rho$, between the fluid inside and outside the bubble can be measured and the surface tension calculated through Laplace's law, $\sigma=\Delta \rho r$, where $\mathrm{r}$ is the radius of the bubble and the difference $\Delta \rho$ is given by the ideal gas law $\Delta \rho=\Delta \rho T$. The surface tension is calculated in this way to be $\sigma=1.8 \times 10^{-2}$. Hence we see that, for the parameters used here:

$$
g^{\prime} k+\left[\frac{k^{3} \sigma}{\rho(f+1)}\right]^{-1} \approx \frac{1}{2}
$$

Thus both surface tension and gravity forces are important in determining the wave motion, although the influence of gravity is less than that of surface tension.

Determination of the interface thickness: This interface thickness can be found by considering the concentration difference, $\mathrm{d}$, which is the difference in the densities of the two fluids. This has an approximately constant positive value in one fluid and an approximately constant negative value of the same magnitude in the other fluid. At the interface $d$ varies smoothly. In each vertical column of simulated data the order parameter was fitted ${ }^{[16]}$ to a curve of the form $d=$ a tanh $(b z+c)$, where $a, b$ and $c$ are the parameters being found by the fitting process.

Since there are many points away from the interface the value $n$ of a should be found very accurate. The values of $b$ and $c$ are determined by about ten points over the interface. This is, however, enough points to determine a good fit with the simulated data. A typical fit is shown in Fig. 1 indicating that the interface has a $\tan \mathrm{h}$ shape and that the results from curve fitting are accurate. The accuracy of the curve fitting can also be seen by considering the vertical position of the center of the interface, where $d=0$. Now for the interface defined as $d=a \tan h(b z+c)$ the center of the interface occurs when $z=-c / b$. Figure 2 shows the position of the interface measured in this manner and the position of the last site above the interface. These results show that the curve fitting procedure gives an accurate method for determining the position of the interface to an accuracy much greater than one lattice site and that the values of $b$ and $c$ are found to a reasonable accuracy despite the relatively small number of sites in the interface region.

The gradient of $\mathrm{d}$ can be easily found by differentiating $d=a \tan \mathrm{h}(b z+c)$, allowing us to find the gradient of the order parameter at the center of the interface: $\left.\frac{\partial d}{\partial z}\right|_{d=0}=a b$.

Now we can define the vertical thickness of the interface:

$$
v=\Delta d\left(\left.\frac{\partial d}{\partial z}\right|_{d=0}\right)^{-1}=\frac{2}{b}
$$

where, $\Delta d$ is the change in d across the interface which is $2 \mathrm{a}$. This definition of $\mathrm{v}$ is chosen since it fits in with our analysis scheme. In other situations a different destination may be preferred, for example the vertical interface thickness might commonly be taken to be v' = $\mathrm{z}_{1}-\mathrm{z}_{2}$ where $\mathrm{d}\left(\mathrm{z}_{1}\right)=0.95 \mathrm{a}$ and $\mathrm{d}\left(\mathrm{z}_{2}\right)=-0.95 \mathrm{a}$. In this case $v^{\prime}=\left[\tanh ^{-1}(0.95)\right] v$ that is the different definitions of the vertical interface thickness will only differ by a constant magnitude term.

Consider a linear interfacial wave with the interface at $\mathrm{z}=\alpha \sin (\mathrm{kx}+\Psi)$ as shown in Fig. 3 At any point $\mathrm{x}_{0}$ the tangent to the interface intersects the horizontal at an angle $\theta$ where $\tan \theta=\alpha \mathrm{k} \sin (\mathrm{kx}+\Psi)$.

Now the interface thickness, $\mathrm{t}$, is given by $\mathrm{t}=\mathrm{v}$ $\cos (\theta)$ or:

$$
t=\frac{2}{b} \cos \left(\tan ^{-1}[\alpha k \cos (k x+\psi)]\right) .
$$

This means that the interface thickness can be found from the fitted parameter $b$ provided the values 
of $\alpha$, the wave amplitude, and $\psi$, the wave phase can be found, the value of $\mathrm{k}$ is known when the wavelength is fixed. Several methods were considered for obtaining these values include estimating them from the position and depth of the crest and the trough and applying the curve fitting algorithm to the surface profile.

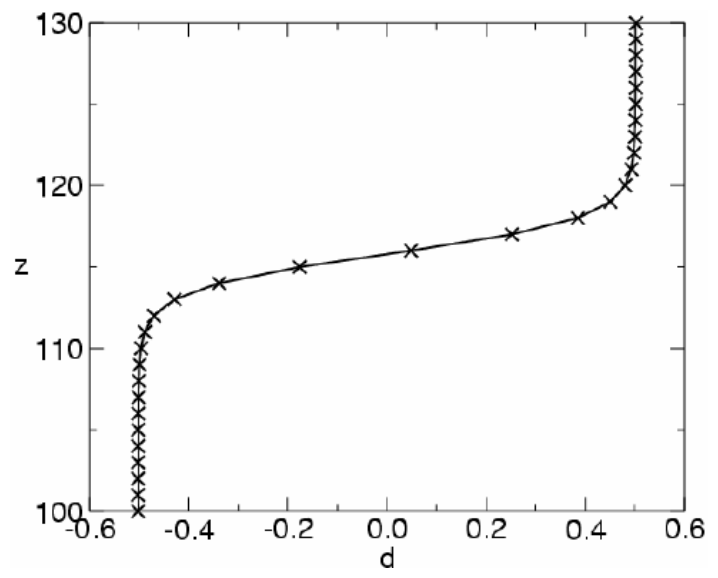

Fig. 1: Comparison, in the Area of the Interface, of the Simulated Order Parameter (Symbols) and the Fitted Tanh Profile

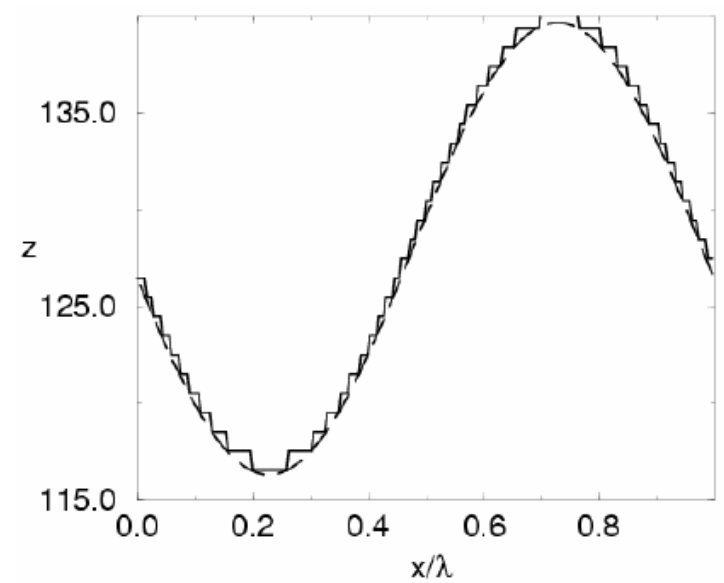

Fig. 2: The Center of the Interface Calculated as $z=-c / b$ (Dashed Line) and as the Last Site above the Interface (Solid Line)

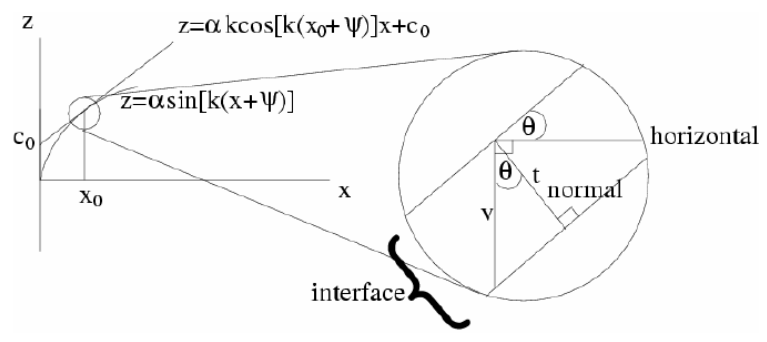

Fig. 3: The Interface Thickness, $t$, the Vertical Thickness, $v$, and the Angle between the Interface and the Horizontal, $\theta$
The method used here, however, was to find the value of $\alpha \mathrm{k} \cos (\mathrm{kx}+\psi)$ rather than the individual variables. This was done by differentiating the central interface profile $(-\mathrm{c} / \mathrm{b})$ using a central difference equation.

\section{RESULTS}

A progressive internal wave was initialized using the method described above for the parameters discussed. Analysis of the position of the interface, $\eta=-c / b$, (scaled by a factor $1 / 30$ ), the vertical thickness, $v=2 / b$, measured from the simulation and the interface thickness:

$$
t=\frac{2}{b} \cos \left(\tan ^{-1}\left[\frac{\mathrm{d}(-c / b)}{\mathrm{d} x}\right]\right)
$$

After 2,000 times-steps. The value of the interface thickness, observed in Fig. 4 is seen to vary by about $4 \%$ depending on the phase of the wave. The two vertical lines are at the crest and the trough of the wave and correspond to the position at which $t$ and $v$ coincide since the interface is horizontal. The peak values of the interface thickness occur slightly behind the crest and the trough of the internal wave. The interface changes from its initial uniform vertical thickness (with $\mathrm{v}$ varying by only $0.05 \%$ in a non-sinusoidal manner) to the approximately seasonal variation (with half the wavelength of the internal wave) in the first 2,000 times-steps. During the next 4,000 times-steps the interface thickness continues to change until it reaches the final from shown in Fig. 5 which shows the thickness of the interface after 8,000 times-steps. The thickness sill peaks just behind the crest and the trough of the wave, however now the peak of the wave crest is larger than the one at the trough. The minimum values of the interface thickness occur between the peaks with the minimum behind the crest being more pronounced than the other minimum. This appears to be the final form of the interface thickness.

The variation in the thickness of the interface is caused by the propagation of the internal wave across the interface. Thus we would expect the variation in the thickness to decay at the same rate as the wave is being damped. The height of the internal wave, $\mathrm{H}$, which is twice the wave amplitude can be calculated as the differences between the maximum height of the interface and the minimum height. Similarly $h$, the height of the variation in interface thickness, can be found as the difference between the mean of the two maximum thicknesses and the mean of the two minimum thicknesses. This is shown in Fig. 6 which shows the rate at which $\mathrm{H}$ and $\mathrm{h}$ are damped. The damping rate of the wave is approximately constant. The best straight-line fit through the results for $H$ was found and a straight line with the same gradient has been drawn through the results for the h. Four times greater than about 8,000 time-steps to be damped at approximately the same rate as the wave. 


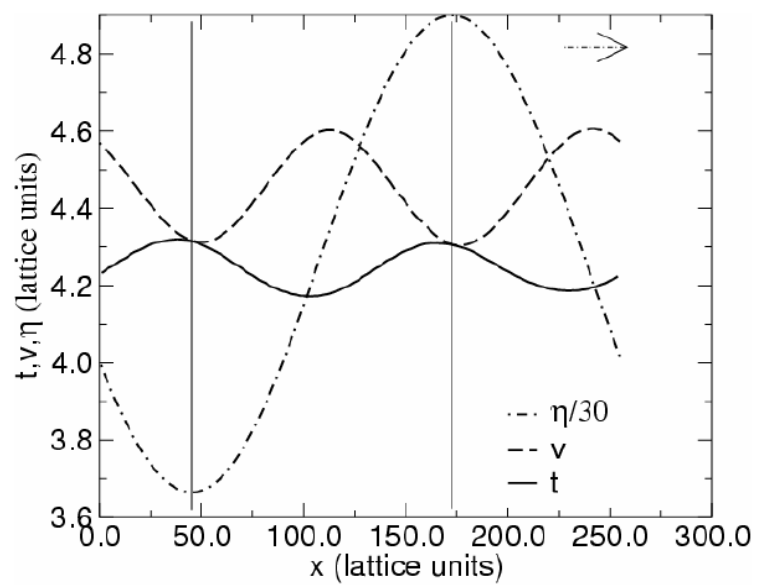

Fig. 4: The Shape of the Interface (Dot-Dashed Line) Travelling from Left to Right, the Measured Vertical Interface Thickness (Dashed Line) and the Calculated Interface Thickness (Solid Line) after 2,000 Time-Steps

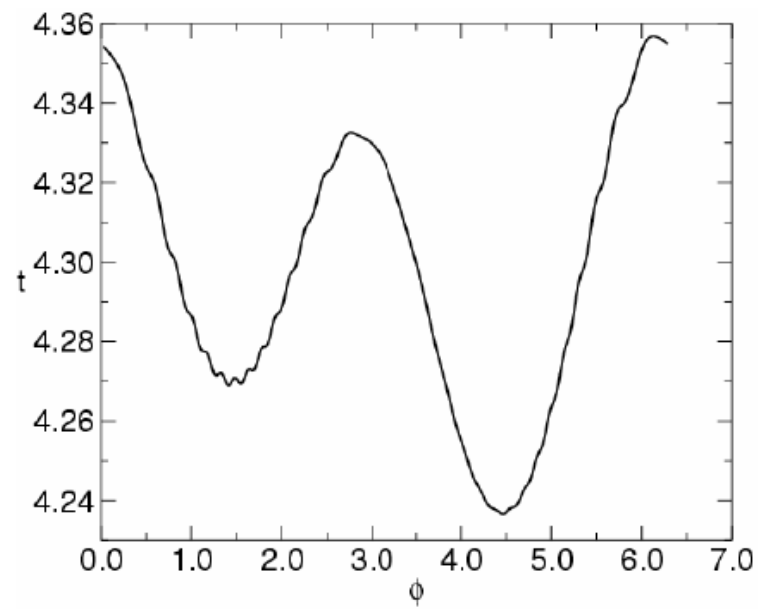

Fig. 5: The Thickness of the Interface after 8,000 Time-Steps as a Function of the Wave Phase, $\phi$, the Crest of the Wave Corresponding to $\phi=0$

At times earlier than 8,000 times-steps there is some deviation probably due to the initial formation of the variations in interface thickness and the changing of these variations as observed between Fig. 4 and 5. The position of the maxima and minima are shown in Fig. 7 relative to the phase of the wave, $\phi=0$ corresponds to the wave crest. In general these occur at $\phi$ slightly less than $n \pi / 2$ for $n=1,2,3,4$ as was observed for the maxima in Fig. 4. Initially the position of the extremes is relatively constant. As the wave decays and the variation in the interface thickness becomes small, the results become noisy making the determination of the exact maximum or minimum impossible, thus the results become less accurate as time increases. This is particularly true for the first maximum and minimum after the wave crest so these results are only shown four times $<32,000$.

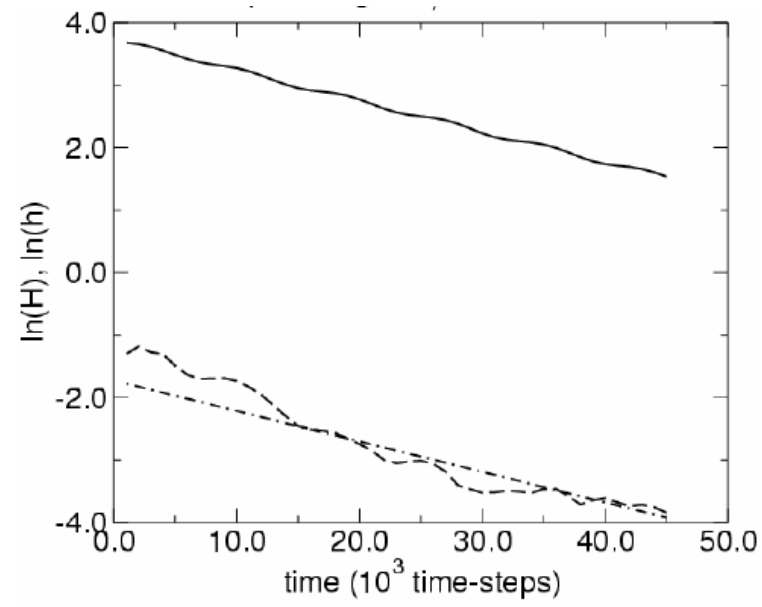

Fig. 6: The Damping of the Wave Height (Solid Line) and the Interface Thickness Variations (Dashed Line) as Functions of Time. Also Shown (DotDash Line) is a Line with the Same Gradient as the Best-Fit Strength Line through the Results for $H$ Drawn through the Results for $h$

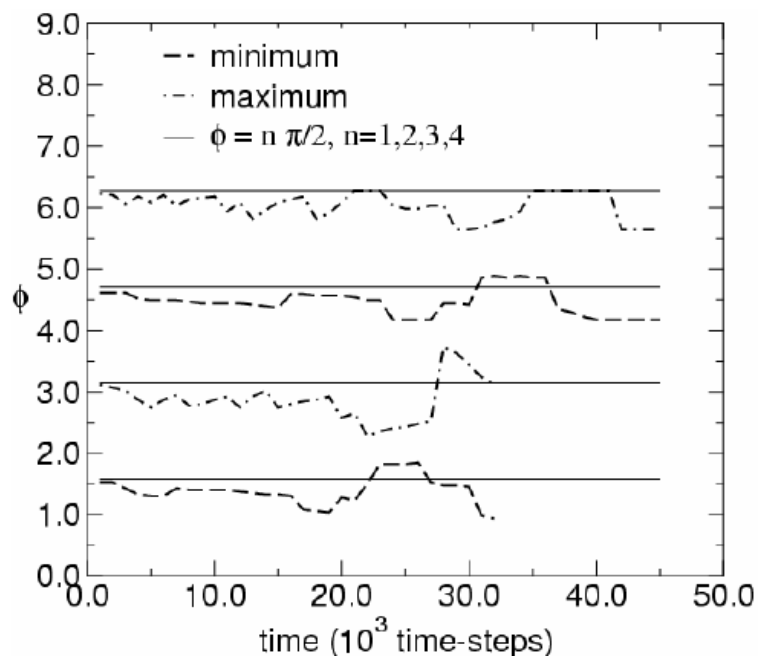

Fig. 7: The Positions of the Maxima and Minima Interface Thickness Relative to the Phase of the Internal Wave, $\phi=0$ Corresponds to the Crest of the Wave

The maximum and minimum behind the crest are larger and so an estimation of their position can be made for $>32,000$ although it will be less accurate that the earlier measurements. The approximately constant position of the maxima and minima relative to the phase of the propagating wave is consistent with the interface thickening and thinning being a direct result of the wave motion.

\section{DISCUSSION}

The results have shown that internal wave propagation at an interface can cause a variation in the 
interface thickness. Consider the following simple model for the interface. Assume that the interface consists of a series of small incompressible elements which do not translate in the $\mathrm{x}$-direction and which have length $\Delta \mathrm{x}$ and width $\mathrm{t}$ when the interface is flat. If the interface is perturbed by an amount $\eta(x)=\alpha \cos$ $(\mathrm{kx}-\Psi)$, then each element is stretched to a length $\Delta s=\left[(\Delta x)^{2}+(\Delta y)^{2}\right]^{1 / 2}$, where $\Delta y=\alpha \cos [k(x+\Delta x)+\psi]-\alpha \cos [k x+\psi]$.

Thus the new length of the element is $\Delta s \approx \Delta x\left[1+\frac{1}{2} \frac{\partial \eta^{2}}{\partial x}(x)\right]$, that is a relative increase in length of $\Delta x^{*}=\frac{1}{2} \frac{\partial \eta^{2}}{\partial x}$. Thus we expect a relative decrease in the thickness of $t^{*}$, where:

$$
t^{*} \approx \frac{1}{2} \alpha^{2} k^{2} \sin ^{2}(k x+\psi)
$$

Comparing the expression for $\mathrm{t}^{*}$ with the results in Fig. 1 and 4, we see from Fig. 4 that the interface thickness has a maximum of 4.33 when $\cos (\mathrm{kx}+\psi) \approx 1$ and $\cos (k x+\psi) \approx-1$, (corresponding to $t^{*} \approx 0$ ). This value of $\mathrm{t}$ is comparable to the value of 4.35 (corresponding to $b=0.46$ ) found from the tanh fit in Fig. 1. The minimum value of the thickness in Fig. 4 occurs close to $\cos (\mathrm{kx}+\psi)=0$. The maximum relative variation in $\mathrm{t}$ is found from Fig. 4 to be $\mathrm{t}_{\text {max }}^{*}=0.036$. The analytic value is $\mathrm{t}_{\text {max }}=0.097$ which is somewhat larger but of the same order of magnitude. This suggests that the observed change in the interface thickness may be due to the interface stretching days' its shape changes. As noted in Fig. 4, there is a small time lag between the wave amplitude and the interface thickness which is not predicted by the simple model represented by above. A time lag is to be expected since the thickness will not change instantaneously with a change in the interface shape. Other factors, such as the velocity shear produced by the wave, may also be contributing to the changes in the interface thickness.

The lattice Boltzmann model utilized here incorporates the correct free energy of a binary fluid to a thermodynamically consistent fluid with a thermodynamically consistent equilibrium state ${ }^{[10]}$. It is therefore well suited to studying capillary waves at the interface. Here the model has been applied in conjunction with a body force to simulate waves where both gravity and surface tension act as the interface. If pure gravity waves were to be considered the nature of the interface will not be significant in determining the wave behavior. In such a case this lattice Boltzmann model has already been shown to give a realistic representation of wave motion ${ }^{[7]}$. Thus we expect the model and the results presented above to be applicable to a wide range of waves extending from pure capillary to pure gravity waves.
The results presented here show a variation in the thickness of a fluid interface as an internal wave propagates along it. The variation can be thought of as being approximately sinusoidal with wavelength half that of the progressive wave, although the two maxima which occur slightly behind the wave crest and trough need not have the same value, and similarly the two minima need not be equal. In a binary system where the two fluids have the same mass any wave propagating at the interface is a pure capillary wave. In such a case inverting the $\mathrm{z}$ direction should have no effect on the system and so we would not expect there to be any differences between the values of the two maximum thicknesses or the two minimum thicknesses. This should also be true if the two fluids have different masses but the wavelength is short enough that the waves can be considered as capillary waves. Thus the differences observed between the interface thickness at the wave peak and the wave trough can be associated with the density difference of the two fluids. It is expected that this difference will decrease with the wavelength and the density difference. As the wavelength is increased or the density difference becomes larger we expect the difference to remain and probably become larger.

In conclusion, we have observed a variation in the thickness of a fluid interface when an internal wave propagates along it. The magnitude of this variation was seen to depend on the wave amplitude in that it is damped at approximately the same rate. The thickness of the interface is maximum slightly behind the wave crest and trough and minimum halfway between the maxima. Some variation was also observed between the two maxima and minima in each wavelength, particularly at larger themes: the maximum just behind the crest being larger than the maximum just behind the trough and the minimum behind the larger maximum being more pronounced than the other minimum.

\section{REFERENCES}

1. Sokolov, S. S., G.Q. Hai and N. Studart, 1997. Damping of interfacial waves in He-3-He-4 layered mixtures Phys. Rev., 56: 8988-8996.

2. Rees, J.M., P.S. Anderson and J.C, King, 1998. Observations of solitary waves in the stable atmospheric boundary layer. Boundary-Layer Meteorol., 86: 47-61.

3. Stanton, T.P. and L.A. Ostrovsky, 1998. Observations of highly nonlinear internal solutions over the Continental Shelf. Geophys. Res. Lett., 25: 2695-2698.

4. Meiron, D.I. and P.G. Saffman, 1983. Overhanging interfacial gravity-waves of large-amplitude. J. Fluid Mech., 129: 213-218.

5. Turner, R.E.L. and J.M. Vanden-Broeck, 1986. The limiting configuration of interfacial gravitywaves. Phys. Fluids, 29: 372-375. 
6. Grimshaw, R.H.J. and D.I. Pullin. 1986. Extreme interfacial waves. Phys. Fluids, 29: 2802-2807.

7. Buick, J.M. and C.A. Greated, 1998. Lattice Boltzmann modeling of interfacial gravity waves. Phys. Fluids, 10: 1490-1511.

8. Buick, J.M., J.A. Cosgrove and C.A. Greated 2004. Gravity-capillary internal wave simulation using a binary fluid lattice Boltzmann model. Applied Mathematical Modelling, 28: 183-195.

9. Orlandini, E., M.R. Swift and J.M. Yeomans, 1995. A lattice Boltzmann model of binary-fluid mixtures. Europhys. Lett., 32: 463-468.

10. Swift, M.R., E. Orlandini, W.R. Osborn and J.M. Yeomans, 1996. Lattice Boltzmann simulations of liquid-gas and binary fluid systems. Phys. Rev. E., 54: 5041-5052.

11. Bhatnagar, P.L., E.P. Gross and M. Krook, 1954. A model for collision processes in gases. I. Small amplitude processes in charged and neutral onecomponent systems. Phys. Rev., 94: 511-525.
12. Noble, D.R., S. Chen, F.G. Georgiadis and R.O. Buxkius, 1995. A consistent hydrodynamic boundary-condition for the lattice Boltzmann method. Phys. Fluids, 7: 203-209.

13. Buick, J.M., A.J. Martin, J.A. Cosgrove, C.A. Greated and W.J. Eassion, 2003. Comparison of a lattice Boltzmann simulation of steep internal waves and laboratory measurements using particle image velocimetry, European J. MechanicsB/Fluids, 22: 27-38.

14. Defant A., 1961. Physical Oceanography, Volume II, Pergamon Press.

15. Jeng, U.S., L. Esibov, L. Crow and A. Steyerl, 1998. Viscosity effect on capillary waves at liquid interfaces. J. Phys. Condens. Matter, 10: 49554962.

16. Gill, P.E. and W. Murray, 1978. Algorithms for the solution of the nonlinear least-squares problem. SIAM J. Numer. Anal., 15: 977-992. 